\title{
The Evolution of Field Spiral Galaxies over the Past 8 Gyr
}

\author{
Asmus Böhm and Bodo L. Ziegler \\ University Observatory Göttingen \\ Geismarlandstr. 11, 37083 Göttingen, Germany \\ boehm@uni-sw.gwdg.de
}

\begin{abstract}
We have performed a large observing campaign of intermediate-redshift disk galaxies including multi-object spectroscopy with the FORS instruments of the Very Large Telescope and imaging with the Advanced Camera for Surveys onboard the Hubble Space Telescope. Our data set comprises 113 late-type galaxies in the redshift range $0.1<z<1.0$ and thereby probes galaxy evolution over more than half the age of the universe. Spatially resolved rotation curves have been extracted and fitted with synthetic velocity fields that account for geometric distortions and blurring effects. With these models, the intrinsic maximum rotation velocity $V_{\max }$ was derived for 73 spirals within the field-of-view of the ACS images. Combined with the structural parameters from twodimensional surface brightness profile fitting, the scaling relations (e.g., the Tully-Fisher Relation) at intermediate redshift were constructed. The evolution of these relations offers powerful tests of the predictions of simulations within the Cold Dark Matter hierarchical scenario.

By comparing our sample to the Tully-Fisher Relation of local spiral galaxies, we find evidence for a differential luminosity evolution: the massive distant galaxies are of comparable luminosity as their presentday counterparts, while the distant low-mass spirals are brighter than locally by up to $>2^{m}$ in rest-frame $B$. Numerous tests applied to the data confirm that this trend is unlikely to arise from an observational bias or systematic errors. Discrepancies between several previous studies could be explained as a combination of selection effects and small number statistics on the basis of such a mass-dependent luminosity evolution. On the other hand, this evolution would be at variance with the predictions from numerical simulations. For a given $V_{\max }$, the disks of the distant galaxies are slightly smaller than those of their local counterparts, as expected for a hierarchical structure growth. Hence, the discrepancy between the observations and theoretical predictions is limited to the properties of the stellar populations. A possible explanation could be the suppression of star formation in low-mass disks which is not yet properly implemented in models of galaxy evolution.
\end{abstract}




\section{Introduction}

Within the last few years, our knowlegde of the basic parameters which determine the past, present and future of the universe has improved significantly. Thanks to the combined results from studies of the Cosmic Microwave Backgrund, the Large Scale Structure, Big Bang Nucleosynthesis and distant supernovae, we now have strong evidence for a flat metric of spacetime (Spergel et al. 2003 and references therein). According to the observations, $73 \%$ of the mean density of the universe originate from Dark Energy, $23 \%$ are contributed by Cold Dark Matter and only $4 \%$ by "ordinary" baryonic matter. In such a cosmology, structure growth proceeds hierarchically, with small structures forming first in the early cosmic stages, followed by the successive build-up of larger structures via merger and accretion events.

Although the constituents of the Dark Energy and Dark Matter remain unknown, the $\Lambda \mathrm{CDM}$ or "concordance" cosmology has been a very successful tool for the interpretation of structures on Mpc scales and beyond (e.g., Peacock 2003). On scales of individual galaxies, however, several discrepancies between observational results and theoretical predictions have been found, a prominent of which is the "angular momentum problem". This term depicts the loss of angular momentum of the baryons to the surrounding DM halo, resulting in galactic disks within numerical simulations which are smaller than observed (e.g., Navarro \& White 1994), however more recent studies made progress in this respect (e.g., Governato et al. 2004). Aiming at a quantitative test of the hierarchical scenario at the scale of individual galaxies, we performed an observational study which covers a significant fraction of the Hubble time.

For this purpose, we utilised scaling relations like the Tully-Fisher relation (TFR, Tully \& Fisher 1977) between the luminosity $L$ and the maximum rotation velocity $V_{\max }$ of spiral galaxies. Basically, this correlation can be understood as a combination of the virial theorem and the rotational stabilisation of late-type galaxies. By comparing local and distant spirals of a given $V_{\max }$, the luminosity evolution within the look-back time can be determined. Since the maximum rotation velocity is a measure for the total (virial) mass of a disk galaxy $\left(V_{\max }^{2} \propto M_{\text {vir }}\right.$, e.g. van den Bosch 2002), the TF analysis relates the evolution of stellar population properties to the depth of the gravitational potential well.

Numerical simulations within CDM-dominated cosmologies have been successfully used to reproduce the observed slope of the local TFR, whereas the zero points were offset due to dark halos with too high concentrations (e.g., Steinmetz \& Navarro 1999). The TFR slope was predicted to remain constant with cosmic look-back time in such $N$-body simulations; nevertheless the modelling of realistic stellar populations at sufficient resolution remains a challenge - typically, the masses of individual particles are of the order of $10^{5} M_{\odot} \ldots 10^{6} M_{\odot}$. Other theoretical approaches focussed more on the chemospectrophotometric aspects of disk galaxy evolution. For example, Boissier \& Prantzos (2001) calibrated their models to reproduce the observed colors 
of local spirals. Compared to these, the authors predicted higher luminosities for massive disks and lower luminosites for low-mass disks at redshifts $z>0.4$. A similiar evolution was found by Ferreras \& Silk (2001). By modelling the mass-dependent chemical enrichment history of disk galaxies with the local TFR as a constraint, the authors found a TFR slope that increases with look-back time (i.e., for a parameterisation $L \propto V_{\max }^{\alpha}, \alpha$ increases with redshift).

In the last decade, many observational studies of the local TFR have produced very large samples with $N_{\text {obj }} \approx 1000$ (e.g. Haynes et al. 1999), not only to derive the slope and scatter with high accuracy, but also to map the peculiar velocity field out to $c z \approx 15000 \mathrm{~km} \mathrm{~s}^{-1}$ (e.g. Mathewson \& Ford 1996). Other groups used spirals, partly with cepheid-calibrated distances, to measure the Hubble constant. For example, Sakai et al. (2000) derived a value of $H_{0}=(71 \pm 4) \mathrm{km} \mathrm{s}^{-1} \mathrm{Mpc}^{-1}$ with this method.

At higher redshifts, robust measurements of rotation velocities become increasingly difficult, which is mainly for two reasons. Firstly, because the objects are very faint. Given a redshift of $z=0.5$, the surface brightness at galactocentric radii of $\sim 3 r_{\mathrm{d}}$ - where the regime of constant rotation velocity is reached - is typically $\mu_{B} \approx 27 \mathrm{mag} \operatorname{arcsec}^{-2}$. Spatially resolved spectroscopy at this level has become feasible just with the generation of $10 \mathrm{~m}-$ class telescopes. The second difficulty coming into play arises from the small apparent sizes of the galaxies, this issue will be described in detail in Sect.4.

A number of samples with $10-20$ objects in the regime $0.25<\langle z\rangle<0.5$ have been observed to estimate a possible evolution in luminosity by comparison to the local TFR. The results of these studies were quite discrepant: e.g. Vogt et al. $(1996,1997)$ found only a modest increase in luminosity of $\Delta M_{B} \approx-0.5^{m}$, whereas Simard \& Pritchet (1998) and Rix et al. (1997) derived a much stronger brightening with $\Delta M_{B} \approx-2.0^{m}$. A more recent study of 19 field spirals by Milvang-Jensen et al. (2003) yielded a value of $\Delta M_{B} \approx-0.5^{m}$ and showed evidence for an increase of this brightening with redshift.

It seems likely that some of these results are affected by the selection criteria. For example, Rix et al. selected blue colors with $(B-R)_{\text {obs }}<1.2^{m}$, Simard \& Pritchet strong [O II] emission with equivalent widths $>20 \AA$, while Vogt et al. partly chose large disks with $r_{\mathrm{d}}>3 \mathrm{kpc}$. The two former criteria prefer late-type spirals, whereas the latter criterion leads to the overrepresentation of large, early-type spirals. Additionally, due to the small samples, all these studies had to assume that the local TFR slope holds valid at intermediate redshifts — we will adress this topic again in Sect. 6]

Based on a larger data set from the DEEP Groth Strip Survey (Koo 2001) with $N \approx 100$ spirals in the range $0.2<z<1.3$, Vogt (2001) found a constant TFR slope and only a very small rest-frame $B$-band brightening of $\leq 0.2^{m}$. On the other hand, in a more recent study based on the same survey, an evolution of the luminosity-metallicity relation both in slope and zero point was observed (Kobulnicky et al. 2003). The authors argued that low-luminosity galaxies probably have undergone a decrease in luminosity 
combined with an increase in metallicity during the last $\sim 8 \mathrm{Gyr}$.

Throughout this article, we will assume the concordance cosmology with $\Omega_{\mathrm{m}}=0.3, \Omega_{\Lambda}=0.7$ and $H_{0}=70 \mathrm{~km} \mathrm{~s}^{-1} \mathrm{Mpc}^{-1}$.

\section{Sample Selection \& Observations}

The sample described here has been selected within the FORS Deep Field (FDF, see Heidt et al. 2003), an $U B g R I J K$ photometric survey covering a sky area of $\sim 6 \times 6 \mathrm{arcmin}^{2}$ near the southern Galactic pole. The imaging was performed with the Very Large Telescope (optical bands) and the New Technology Telescope (Near Infrared bands). Based on a catalogue with spectral types and photometric redshift estimates (Bender et al. 2001), we chose objects for follow-up spectroscopy which satisfied the following criteria: 1) late-type Spectral Energy Distribution, i.e., galaxies with emission lines, 2) total apparent $R$-band magnitude $R \leq 23^{m}$, 3) photometric redshift $z_{\text {phot }} \leq 1.2$ to ensure that at least the [O II] 3727 doublet falls within the wavelength range of the spectra, 4) disk inclination angle $i \geq 40^{\circ}$ and 5) deviation between slit direction and apparent major axis of $\delta \leq 15^{\circ}$. The two latter constraints were chosen to limit the geometric distortions of the observed rotation curves. For some objects, however, these limits had to be exceeded during the construction of the spectroscopic setups.

After a pilot observation in 1999, the spectroscopy was performed in 2000 and 2001 using the FORS1 \& 2 instruments of the VLT in multi-object spectroscopy mode with a total integration time of $2.5 \mathrm{hrs}$ per setup. Using the grism $600 \mathrm{R}$, a spectral resolution of $R \approx 1200$ was achieved with a spectral scale of $1.07 \AA /$ pix and a spatial scale of $0.2 \mathrm{arcsec} / \mathrm{pix}$. The seeing ranged between 0.4 and 1.0 arcsec with a median of 0.74 arcsec. In total, 129 late-type galaxies were observed.

For an accurate derivation of the galaxies' structural parameters, like disk inclination, scale length etc., we also took Hubble Space Telescope images of the FDF with the Advanced Camera for Surveys using the F814W filter. To cover the complete FDF area, a $2 \times 2$ mosaic was observed.

\section{Spectrophotometric Analysis}

The spectra of 113 galaxies were reliable for redshift determination. Out of these, 73 objects eventually yielded maximum rotation velocities (see next section) and were covered by the HST/ACS imaging; these objects will be referred to as the FDFTF sample in the following. They span the redshift range $0.09<z<0.97$ with a median of $\langle z\rangle=0.45$ corresponding to look-back times $1.2 \mathrm{Gyr}<t_{1}<7.6 \mathrm{Gyr}$ with $\left\langle t_{1}\right\rangle=4.7 \mathrm{Gyr}$. This data set covers all spectrophotometric types from very early-type spirals (Sa or $T=1)$ to very late-type galaxies ( $\mathrm{Sdm} / \mathrm{Im}$ or $8 \leq T \leq 10)$.

An analysis of the galaxies' surface brightness profile profiles was conducted with the GALFIT package (Peng et al. 2002). To fit the disk com- 
ponent, an exponential profile was used, while a potential bulge was approximated with a Sérsic profile. In the case of 13 FDFTF galaxies, the fit residual images and large fit errors indicated an irregular component that could not be approximated properly with a Sérsic law. The bulge-to-total ratios of these galaxies were assumed to be undefined. The $B / T$ ratios of the other 60 FDFTF galaxies $(0 \leq B / T \leq 0.53$ with $\langle B / T\rangle=0.04)$ confirm that the vast majority of these galaxies are disk-dominated.

Total apparent magnitudes were determined using the mag_auto algorithm of the Source Extractor package (Bertin \& Arnouts 1996). For the computation of absolute $B$-band magnitudes $M_{B}$, we used the filter which, depending on the redshift of a given object, best matched the rest-frame $B$-band. For galaxies at $z \leq 0.25,0.25<z \leq 0.55,0.55<z \leq 0.85$ and $z>0.85$, we thus utilised the $B, g, R$ and $I$ magnitudes, respectively. Thanks to this strategy, the $k$-correction uncertainties $\sigma_{k}$ - usually a substantial source of error to the luminosities of distant galaxies - are smaller than $0.1^{m}$ for all types and redshifts in our sample. For the correction of intrinsic dust absorption, we followed the approach of Tully \& Fouqué (1985) assuming a face-on $\left(i=0^{\circ}\right)$ extinction of $A_{B}=0.27^{\mathrm{m}}$. The absolute magnitudes of the FDFTF galaxies computed this way span the range $-18.0^{m} \geq M_{B} \geq-22.7^{m}$.

The spectra of 12 objects in our sample cover a wavelength range that simultaneously shows emission in [O II]3727, H $\beta$, [O III]4959 and [O III]5007 at sufficient signal-to-noise to determine the equivalent widths. These lines can be used to estimate the gas-phase metallicity. We adopted the analytical expressions given by McGaugh (1991) to compute the abundances $\mathrm{O} / \mathrm{H}$ from the $R_{23}$ and $O_{32}$ parameters. Since all the galaxies have $M_{B}<-18^{m}$, we assumed that they fall on the metal-rich branch of the $R_{23}-\mathrm{O} / \mathrm{H}$ relation. The galaxies have abundances $8.37<\log (\mathrm{O} / \mathrm{H})<8.94$. We will use these estimates to investigate the luminosity-metallicity relation in Sect. 5 .

\section{Derivation of $\mathrm{V}_{\max }$}

We extracted spatially resolved rotation curves from the two-dimensional spectra by fitting Gaussians to the emission lines stepwise along the spatial axis. Line fits at any projected radius which, compared to the instrumental broadening $\left(\mathrm{FWHM}_{\mathrm{ins}} \approx 4.5 \AA\right)$, had very small $\left(\mathrm{FWHM}_{\mathrm{fit}}<2 \AA\right)$ or very large $\left(\mathrm{FWHM}_{\mathrm{fit}}>12 \AA\right)$ line widths were assumed to be noise and therefore neglected.

The analysis of spatially resolved rotation curves from optical spectroscopy of local spiral galaxies is relatively straightforward. But in the case of distant galaxies with very small apparent sizes, the effect of the slit width on the observed rotation velocities $V_{\text {rot }}(r)$ must be considered. At redshift $z=0.5$, a scale length of $3 \mathrm{kpc}$ - typical for an $L^{*}$ spiral - corresponds to $\sim 0.5$ arcsec only, which is half the slit width used in our observations. Any value of $V_{\text {rot }}(r)$ is therefore an integration perpendicular to the spatial axis (slit direction), a phenomenon which is the optical equivalent to "beam smearing" in radio 


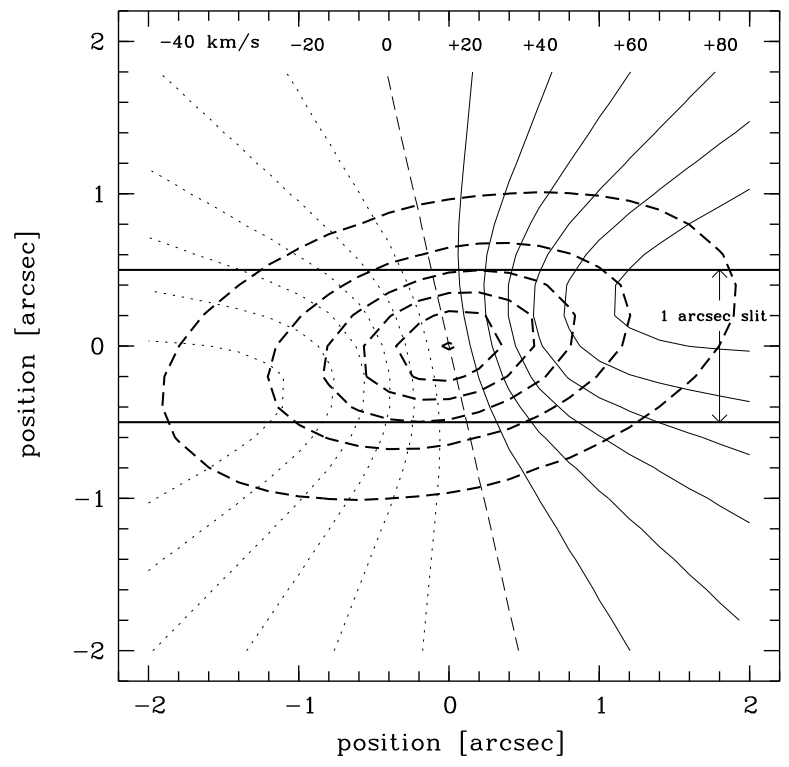

Figure 1: Example of a simulated rotation velocity field for an object from our data set with a disk inclination $i=64^{\circ}$ and a misalignment angle between apparent major axis and slit direction of $\delta=+13^{\circ}$. The dashed ellipses denote the isophotes of the disk, with the outermost corresponding to an $I$ band surface brightness of $\mu_{I} \approx 25 \mathrm{mag} \operatorname{arcsec}^{-2}$. The curved dotted and solid lines correspond to line-of-sight rotation velocities ranging from $-120 \mathrm{~km} / \mathrm{s}$ to $+120 \mathrm{~km} / \mathrm{s}$. The two solid horizontal lines visualise the position of the slit used for spectroscopy.

observations. The seeing has an additional blurring effect on the observed rotation curves. If not taken into account, these two phenomena would lead to an underestimation of the intrinsic rotation velocities and, in particular, the intrinsic $V_{\max }$.

We overcame this problem by generating synthetic rotation curves. For the intrinsic rotational law, we used a simple shape with a linear rise of $V_{\text {rot }}(r)$ at small radii, turning over into a region of constant rotation velocity where the Dark Matter halo dominates the mass distribution. Alternatively, we also tested the so-called "Universal Rotation Curve" shape (Persic et al. 1996), a parameterisation which introduces a velocity gradient in the outer regions of the disk which is positive for sub- $L^{*}$ objects and negative for objects much more luminous than $L^{*}$. However, the results given here are not sensitive to the form of the intrinsic rotational law — see Böhm et al. (2004) for a detailed discussion of this topic - and we therefore only use $V_{\max }$ values determined with an intrinsic "rise-turnover-flat" shape here.

Given the observed inclination, position angle and scale length of an object, the intrinsic rotation velocity field was constructed, Fig. 1 1 shows an example. 

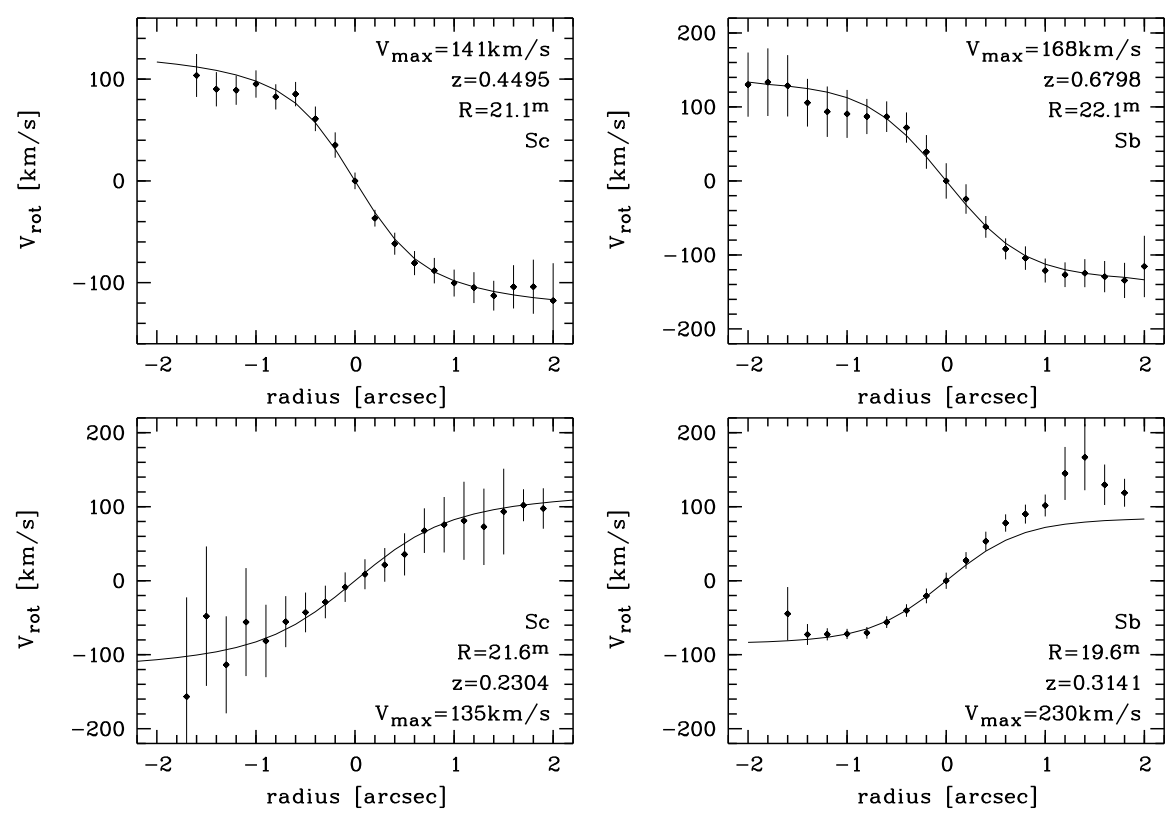

Figure 2: Examples of rotation curves from our data set. The solid lines are the synthetic rotation curves fitted to the observed rotation velocity as a function of radius (solid symbols) used to derive the intrinsic maximum rotation velocity. For each object the spectrophotometric type, total apparent $R$ magnitude, redshift and $V_{\max }$ are given. The two upper curves were classified as high quality data, the two lower ones as low quality data due to the large measurement errors (lower left) or an asymmetric shape (lower right).

In the next step of the simulation, the velocity field was weighted with the surface brightness profile. The effect of this was that, just like for the observed data, brighter regions contributed stronger to the rotation velocities in direction of dispersion than fainter regions (the "beam smearing" effect). Following the weighting, the velocity field was convolved with the Point Spread Function to simulate the blurring due to seeing. Finally, a "stripe" was extracted from the velocity field, with a position and width that corresponded to the slit used during the observations, and integrated perpendicular to the spatial axis. The results of the whole procedure was a synthetic rotation curve which introduced the same geometric and blurring effects as the corresponding observed rotation curve. By fitting the simulated rotation curve to the observed rotation curve, we derived the intrinsic value of $V_{\max }$. Four examples of observed rotation curves along with the best-fitting synthetic rotation curves are shown in Fig. 2

36 galaxies had to be rejected from the further analysis because the $S / N$ was too low to probe the regime of constant rotation velocity at large radii, or because the rotation curves were perturbated. Four objects were reliable 


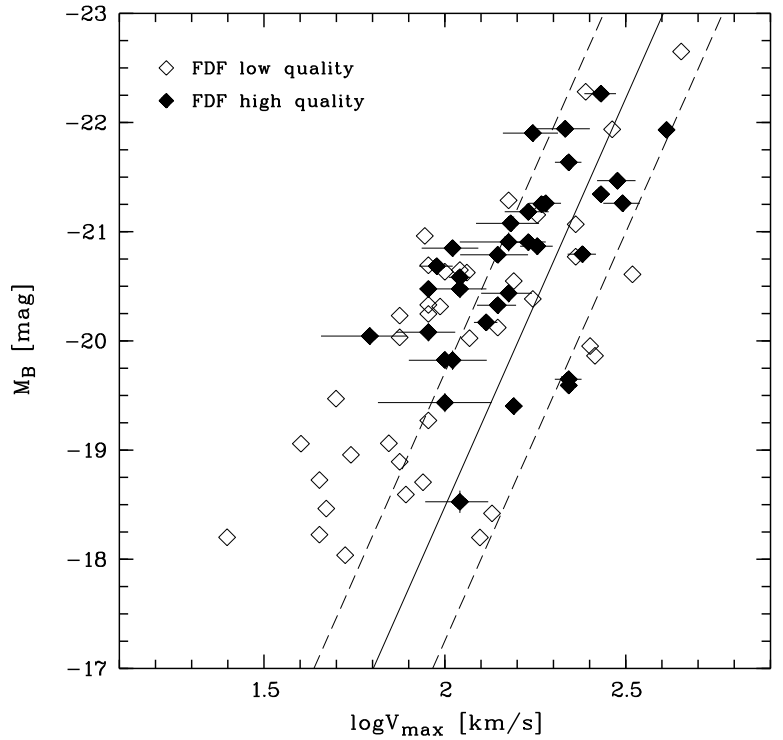

Figure 3: FORS Deep Field sample of spirals in the range $0.1 \leq z \leq 1.0$ in comparison to the local Tully-Fisher relation by Pierce \& Tully (1992); the solid line denotes the fit to the local data, the dashed lines give the $3 \sigma$ limits. The distant sample is subdivided according to rotation curve quality: high quality curves (solid symbols) extend well into the region of constant rotation velocity at large radii and therefore give robust values of $V_{\max }$. Error bars are shown for the high quality data only.

for the $V_{\max }$ determination, but their positions were located outside the fieldof-view of the HST/ACS mosaic imaging. 34 objects had curves with a high degree of symmetry and clearly reached into the "flat" regime, we consider these as high quality data. 39 curves had a relatively small spatial extent or mild asymmetries, these will be referred to as low quality data in the following. In total, our kinematic data set thus comprises 73 late-type galaxies at a mean look-back time of $\sim 5$ Gyr. The objects span the range $25 \mathrm{~km} / \mathrm{s} \leq V_{\max } \leq$ $450 \mathrm{~km} / \mathrm{s}$ with a median of $129 \mathrm{~km} / \mathrm{s}$ (high quality data only: $62 \mathrm{~km} / \mathrm{s} \leq$ $V_{\max } \leq 410 \mathrm{~km} / \mathrm{s}$ and $\left.\left\langle V_{\max }\right\rangle=154 \mathrm{~km} / \mathrm{s}\right)$.

\section{Scaling Relations at Intermediate Redshift}

In Fig. 3. the maximum rotation velocities and absolute magnitudes of the distant FDFTF galaxies are compared to the local $B$-band Tully-Fisher relation by Pierce \& Tully (1992):

$$
M_{B}=-7.48 \log V_{\max }-3.52
$$


with a scatter of $\sigma_{B}=0.41^{m}$. Note that, at variance with the original relation given by these authors, we have calibrated the zero point to a face-on extinction of $0.27^{m}$ to achieve consistency with the computation of the distant galaxies' absolute magnitudes. We emphasize that the further analysis is not sensitive to the choice of the local reference sample: e.g., for the large data set of Haynes et al. (1999, comprising 1097 objects), we find a very similar relation of

$$
M_{B}=-7.85 \log V_{\max }-2.78,
$$

using a bisector fit (two geometrically combined least-square fits with the dependent and indepedent variable interchanged). We will utilise the Pierce \& Tully sample here for the sake of comparability to intermediate-redshift TF studies in the literature which mostly have used this sample as a local reference.

On the average, the distant galaxies are overluminous with respect to their local counterparts, we find a median offset of $\left\langle\Delta M_{B}\right\rangle=-0.98^{m}$ for the total FDFTF sample and $\left\langle\Delta M_{B}\right\rangle=-0.81^{m}$ for the high quality data only. But we find also evidence for a differential evolution. Fig. 3 indicates a relatively good agreement between the intermediate-redshift galaxies and the local TFR in the regime of fast rotators, i.e. high masses, while the distant low-mass galaxies systematically deviate from the relation of present-day spirals. For low quality data, this may partly be due to underestimated maximum rotation velocities, since the corresponding curves have a relatively small spatial extent and do not robustly probe the region of constant rotation velocity at large radii. In the case of high quality rotation curves, this is however unlikely, since these extent well into the "flat region".

A 100 iteration bootstrap bisector fit (average of 100 bisector fits with randomly removed objects in each iteration) to the 34 FDFTF objects with high quality rotation curves yields

$$
M_{B}=-(4.05 \pm 0.58) \log V_{\max }-(11.8 \pm 1.28),
$$

i.e. the TFR slope we find at intermediate redshift is significantly shallower than in the local universe. Since the derivation of the galaxies' structural parameters and of the $V_{\max }$ values has been based entirely on HST/ACS imaging, Eq. 3 is a confirmation of the results presented in Böhm et al. (2004) which were limited to ground-based imaging.

We show the individual offsets $\Delta M_{B}$ of the FDF galaxies from the local TFR as a function of their maximum rotation velocity in Fig. 4. Even when restricting the sample to the high quality rotation curves, we find significant overluminosties of up to more than $2^{m}$ in the rest-frame $B$ for low-mass spirals. $L^{*}$ galaxies, corresponding to $\log V_{\max } \approx 2.3$ according to Eq. 11 scatter around a negligible evolution, while the fastest rotators are systematically underluminous.

In Fig. 5e show the sub-sample of $12 \mathrm{FDF}$ galaxies for which we could determine the oxygen abundances $\mathrm{O} / \mathrm{H}$ in comparison to the local luminositymetallicity relation as given in Kobulnicky et al. (2003, the displayed scatter 


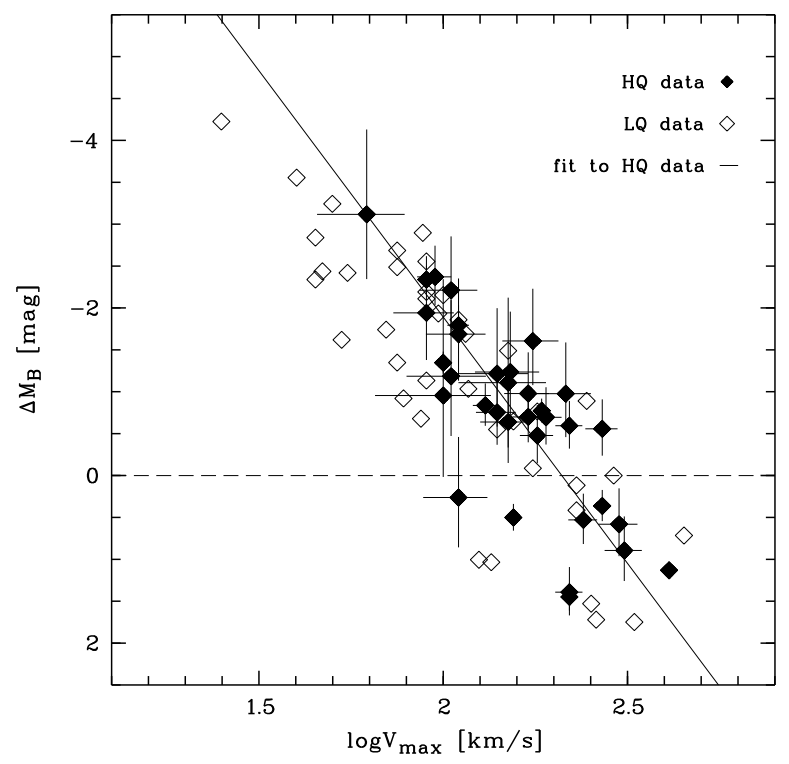

Figure 4: Offsets of the distant FORS Deep Field galaxies from the local TFR by Pierce \& Tully (1992). The distant sample is subdivided according to rotation curve quality: high quality curves (solid symbols) extend well into the region of constant rotation velocity at large radii and therefore give robust values of $V_{\max }$. The dashed horizontal line corresponds a zero luminosity evolution. While high-mass galaxies are in agreement with the local TFR or even slightly underluminous given their $V_{\max }$, the objects are increasingly overluminous towards small values of $V_{\max }$ (error bars are shown for the high quality data only).

is a rough estimate). In addition, a sub-sample of distant late-type galaxies from the DEEP survey (ibid.) is shown, which has been restricted to the same redshift intervall $(0.22<z<0.46)$ that is covered by the FDF galaxies. Both sub-samples thus represent a look-back time of $\sim 4$ Gyr. For the sake of comparability, the absolute magnitudes $M_{B}$ of the FDF galaxies given in this figure are not corrected for intrinsic absorption, as is the case for the Kobulnicky et al. data. Both distant samples indicate a "tilt" with respect to the local $L-Z$ relation. At given $\log (\mathrm{O} / \mathrm{H})$, high-metallicity galaxies at intermediate redshift agree relatively well with the local $L-Z$ relation, whereas low-metallicity objects are overluminous. Alternatively, the distributions may be interpreted such that the distant low-luminosity galaxies have smaller chemical yields than locally, while high-luminosity galaxies do not differ strongly in $\mathrm{O} / \mathrm{H}$ between intermediate and low redshift. If the offsets of the FDF spirals we observe in the TF diagram are due to younger stellar populations than locally, it is probable that Fig. [5 shows a combined evolution in luminosity and metallicity. This indeed has been the conclusion 


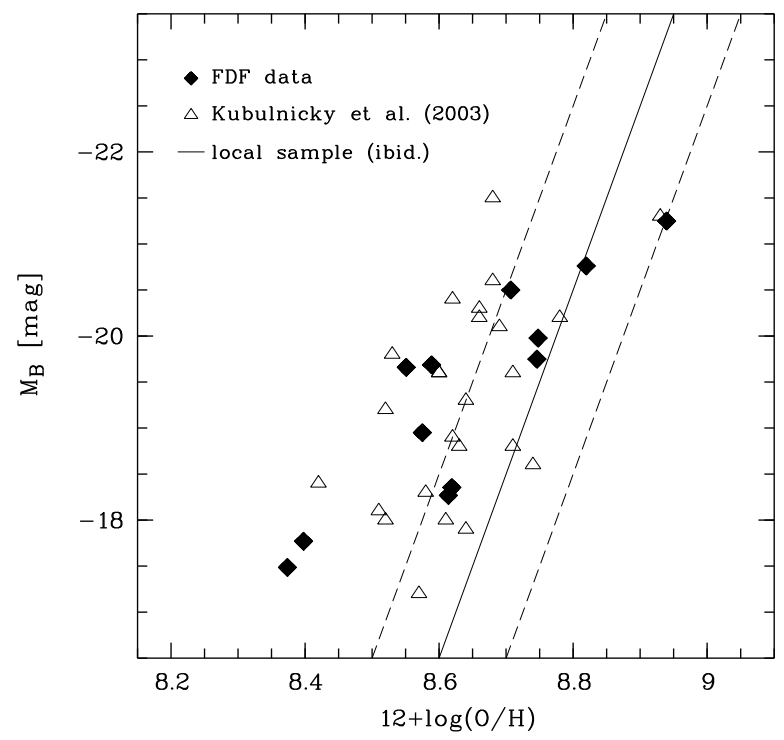

Figure 5: The gas-phase metallicities of 12 spirals from the FDF data set (filled symbols) in comparison to the local luminosity-metallicity relation constructed by Kobulnicky et al. (2003, solid line; dashed lines give the estimated $1 \sigma$ scatter). Also shown are distant galaxies presented in Kobulnicky et al. (open symbols) which cover a similar redshift range as the FDF galaxies. Both distant samples show a "tilt" with respect to the local relation which likelywise indicates a combined evolution in luminosity and metallicity of low-luminosity galaxies.

of Kobulnicky et al. after comparison of their data to single-zone models.

The third scaling relation which we want to focus on here is presented in Fig. 6] where the FDF galaxies are shown with respect to the local velocitysize relation correlating $V_{\max }$ and $r_{\mathrm{d}}$. To derive the latter, we used the sample of Haynes et al. (1999). A bisector fit this data set yields

$$
\log r_{\mathrm{d}}=1.35 \log V_{\max }-2.41 .
$$

Since the scale lengths of the FDF galaxies were determined in the $I$-band (HST-filter F $814 \mathrm{~W}$ ), the data intrinsically probe shorter wavelengths towards higher redshifts. For direct comparability to the local sample ( $I$-band as well), we had to account for this rest-frame shift. Otherwise, the measured FDF disk sizes could be overestimated, in particular for the more distant galaxies. Adopting the relations between scale lengths of local spirals at different wavelengths presented in de Jong (1996), we have transformed the observer's frame $I$-band scale lengths to the rest-frame $I$-band values. Note, however, that these factors are relatively small: for the FDF galaxies at $z \approx 1$, the correction corresponds to only $\sim 10 \%$. The characteristic disk sizes of the FDFTF sample cover the range $0.7 \mathrm{kpc} \leq r_{\mathrm{d}} \leq 10.1 \mathrm{kpc}$ with a median $\left\langle r_{\mathrm{d}}\right\rangle=2.7 \mathrm{kpc}$. 


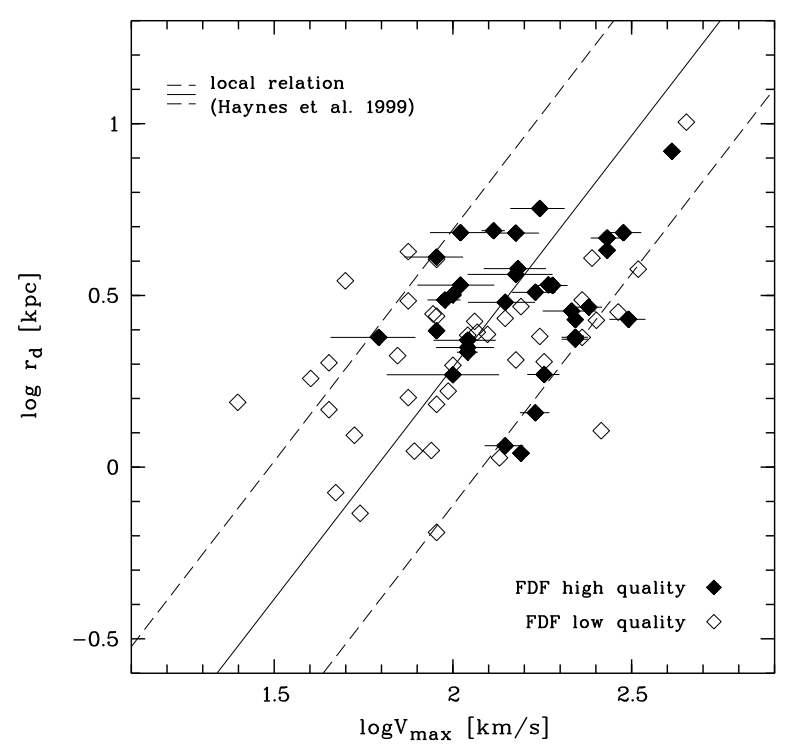

Figure 6: Comparison between the intermediate-redshift FDF galaxies and the local velocity-size relation of the Haynes et al. sample (1999, solid line; dashed lines correspond to the $3 \sigma$ scatter). Error bars are shown for high quality data only.

\section{Discussion}

The $V_{\text {max }}$-dependent TF offsets we observe at redshift $\langle z\rangle \approx 0.5$ may be indicative for a significant decrease of the luminosity of low-mass galaxies possibly combined with an increase in metallicity - over the past $\sim 5 \mathrm{Gyr}$ and a negligible evolution of high-mass galaxies. This evolution would be at variance with theoretical predictions: e.g., Steinmetz \& Navarro (1999) find massindependent TF offsets towards higher redshifts with an $N$-body Smoothed Particle Hydrodynamics code. Boissier \& Prantzos (2001), who used a "backwards approach" model calibrated to the observed chemo-spectrophotometric properties of local spirals, predict overluminosties of high-mass spirals and underluminosities of low-mass spirals towards larger look-back times.

It has to be ruled out that our result might be induced by an observational bias or a systematic error. E.g., it is known that present-day spirals show a correlation between their TF residuals and broad-band colors (e.g. Kannappan et al. 2002), with blue galaxies preferentially populating the regime of overluminosities. We have therefore tested whether Fig. 4 may simply reflect an evolution of the color-residual relation with redshift, finding no evidence for such a trend (Böhm et al. 2004). Another issue that has to be adressed is the potential impact of sample incompleteness. Any magnitude-limited data set contains only a fraction of the objects that are located within the observed volume. Towards lower luminosities (or slower rotation velocities), this frac- 
tion becomes smaller. Furthermore, the magnitude limit corresponds to higher luminosities at higher redshifts. An incompleteness bias could therefore result in a flattening of the distant TFR with increasing redshift. However, dividing our sample into objects with $z \leq 0.449$ (37 galaxies) and $z>0.449$ (36 galaxies), we find no evidence for such a redshift dependence, the respective slopes of the two redshifts bins are -3.49 and -3.77 . For a more sophisticated test of sample incompleteness which is based on the work of Giovanelli et al. (1997), we refer to our results presented in Ziegler et al. (2002).

In the following, we will address another three examples of tests we performed. These are related to the influence of the intrinsic rotation curve shape, the impact of the intrinsic absorption correction and the issue of galaxy-galaxy interactions.

To derive the intrinsic maximum rotation velocity, we have assumed an intrinsic rotation curve shape with a linear rise of the rotation velocity at small radii which turns over into a region of constant rotation velocity at a radius that depends on the rest-frame wavelength of the used emission line. This shape is observed for kinematically unperturbated, massive $\left(\sim L^{*}\right)$ local spirals (e.g., Sofue \& Rubin 2001). For galaxies of very high or very low masses, on the other hand, it is observed that even in the outer parts, most rotation curves have a velocity gradient. While the rotation velocity keeps rising beyond the "turnover" radius in very low-mass spirals, the velocity gradient in very high-mass spirals is negative. Persic et al. (1996) have used $>1000$ curves of local spirals to derive a parameterisation that uses the luminosity of an object as an indicator for the rotation curve shape. To ensure that the observed TF offsets cannot be attributed to a false assumption on the intrinsic rotational law, we have alternatively used this so-called "Universal Rotation Curve" shape as input for the computation of our synthetic velocity fields. If we use the $V_{\max }$ values derived this way to recompute the offsets from the local TFR, the luminosity evolution we find is smaller by only $\sim 0.15^{m}$ at $V_{\max } \approx 100 \mathrm{~km} / \mathrm{s}$. Since this is a very modest change of the offsets we found on the basis of the simple "rise-turnover-flat"-shape (which have a median of $\left\langle\Delta M_{B}\right\rangle \approx-1.74^{m}$ at $V_{\max } \approx 100 \mathrm{~km} / \mathrm{s}$ for the HQ data), we conclude that our results do not differ significantly between these two assumptions on the intrinsic rotation curve shape.

Similarly, we have tested whether a different approach to correct for the intrinsic absorption would have an effect on our results. All values given here were derived following Tully \& Fouqué (1985), i.e., the amount of intrinsic absorption is assumed to depend only on the inclination of the disk. More recently, Tully et al. (1998) have found evidence that the dust reddening is - at least locally - stronger in high-mass spirals than in low-mass spirals. Using their results, we have recomputed the absolute magnitudes of the FDFTF galaxies. As a new local reference that is consistently corrected for intrinsic absorption following Tully et al., we adopted the sample of Verheijen (2001) which is slightly steeper (slope -8.1) than the Pierce \& Tully (1992) sample. This is simply due to the fact that fast rotating, high-mass spirals are assumed to have a larger amount of intrinsic absorption than in the Tully \& Fouqué 
approach, and vice versa in the low-mass regime. The offsets of the FDF high quality data from the Verheijen TFR are however very similar to the initial values $\left(\left\langle\Delta M_{B}\right\rangle \approx-1.77^{m}\right.$ vs. $\left\langle\Delta M_{B}\right\rangle \approx-1.74^{m}$ at $\left.V_{\max } \approx 100 \mathrm{~km} / \mathrm{s}\right)$. With respect to the two conventions of intrinsic absorption correction discussed here, the TF offsets therefore are robust.

A third aspect we want to focus on here concerns the interplay between the stellar population properties and the environment. From studies in the local universe, it is known that galaxies residing in close pairs can be subject to tidal interactions which can increase the star formation rates. In such cases, the fraction of high-mass stars would be enlarged and, in turn, the mass-to-light ratio would be decreased, resulting in overluminosities in the TF diagram. This triggering of star formation would be particularly efficient in low-mass galaxies (e.g., Lambas et al. 2003). Though we have selected our objects from a sky region that should be representative for low-density environments, it is not clear a priori whether the correlation between the TF offsets and $V_{\max }$ can at least in part be attributed to tidally induced star formation. Based on $>10^{5}$ galaxies from the $2 \mathrm{dF}$ survey, Lambas et al. have found that the star formation rates can be significantly increased in objects that have close companions with a separation $\Delta V_{\text {sys }} \leq 250 \mathrm{~km} / \mathrm{s}$ in systematic velocity and a projected distance of $D_{\text {proj }} \leq 100 \mathrm{kpc}$. Using all 267 available spectroscopic redshifts of FDF galaxies at $z \leq 1$ from our own study and a low-resolution survey presented in Noll et al. (2004), and adopting the Lambas et al. constraints cited above, we have found 12 FDFTF objects with confirmed neighbors.

In Fig. 7 we show the TF offsets of these galaxies in comparison to the rest of the sample. Though the small sub-sample of pair candidates does not allow robust statistics, the galaxies with close companions appear to be similarly distributed as the rest of the sample. Moreover, we find a hint that the rotation curve quality is reduced with respect to the probably isolated galaxies - only 3 out of $12(25 \%)$ pair candidates were classified to have high quality rotation curves, whereas for the rest of the sample, this fraction is 31 out of 61 (51\%). In particular, the pair candidates with HQ curves are not systematically biased towards large overluminosities. Since we have spectroscopic redshifts only for a fraction of the galaxies within the probed volume, it is possible that we missed some close pairs. However, the aim was to test whether those pair candidates which are identified systematically differ from the remaining FDFTF objects. We thus conclude that our analysis which is based on the high quality rotation curves is very unlikely to be affected by tidally induced star formation.

To summarise all the tests performed, we find no evidence for any systematic error or bias that may be the source of the observed shallow slope of the intermediate-redshift TFR. We now will show that our findings can be used to interpret the rather discrepant results of previous TF studies of distant galaxies introduced in Sect. 1] For this, Fig. 8] shows our sample sub-divided according to the Spectral Energy Distribution into early-type spirals ( $\mathrm{Sa} / \mathrm{Sb})$, intermediate-types (Sc) and very late-types (Sdm/Im). All three sub-samples 


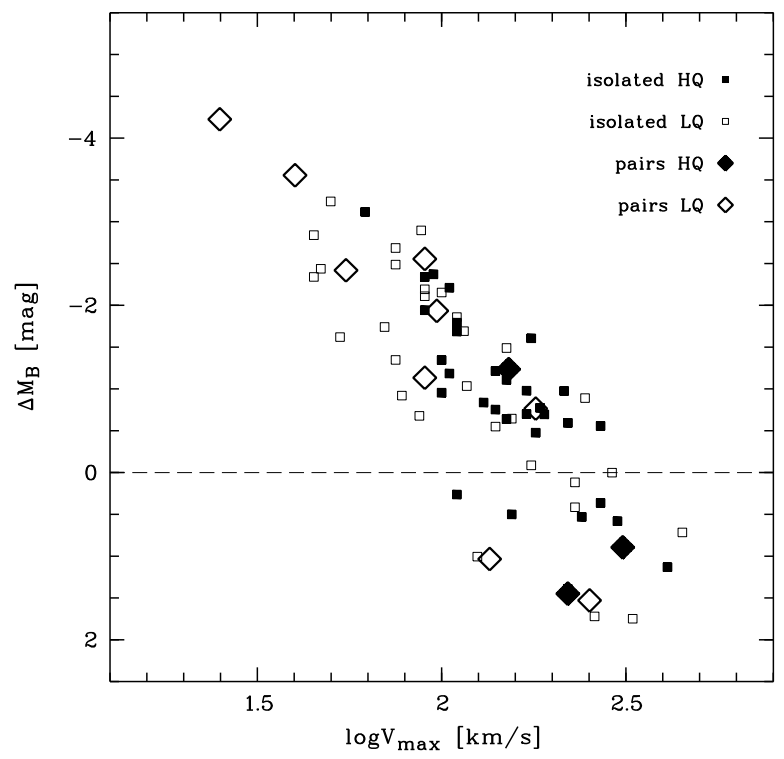

Figure 7: Offsets of the distant FORS Deep Field galaxies from the local TFR by Pierce \& Tully (1992). Large symbols depict FDF objects which show spectroscopically confirmed neighbors within $\Delta V_{\text {sys }} \leq 250 \mathrm{~km} / \mathrm{s}$ in systematic velocity and a projected separation $D_{\text {proj }} \leq 100 \mathrm{kpc}$. See text for details.

show a correlation between the TF offsets and $V_{\max }$, but cover different mass regimes: galaxies with late-type spectra have smaller average $V_{\max }$ values than early-type spirals. The respective classes have median values of $\left\langle V_{\max }\right\rangle_{\mathrm{Sdm} / \mathrm{Im}}=91 \mathrm{~km} / \mathrm{s},\left\langle V_{\max }\right\rangle_{\mathrm{Sc}}=140 \mathrm{~km} / \mathrm{s}$ and $\left\langle V_{\max }\right\rangle_{\mathrm{Sa} / \mathrm{Sb}}=240 \mathrm{~km} / \mathrm{s}$. For samples which are too small to robustly test a correlation between $\Delta M_{B}$ and $V_{\max }$ but can only be used to determine average TF offsets, this would results in a correlation between SED type and $\left\langle\Delta M_{B}\right\rangle$.

Studies comprising only 10-20 galaxies which were selected on blue colors or strong emission lines and therefore preferentially contained late-type spirals (e.g., Rix et al. 1997, Simard \& Pritchet 1998), found evidence for large luminosity offsets at intermediate redshift with respect to the local TFR. According to the above, this can be attributed to a combination of a selection effect and small number statistics. Similary, a study by Vogt et al. (1996) which was selected on large disks and therefore mainly contained early-type spirals (which on average are more massive than late-types), yielded a modest value for the mean $\mathrm{TF}$ offset.

A more recent study on $\sim 100$ intermediate-redshift spirals by Vogt (2001) did not find evidence for a slope evolution of the TFR, at variance with our results. Moreover, this study yields only a modest average TF offset of $-0.2^{m}$, compared to the $-0.8^{m}$ we find for the HQ data (note that these values correspond to the same cosmology and similar redshifts). It is difficult 


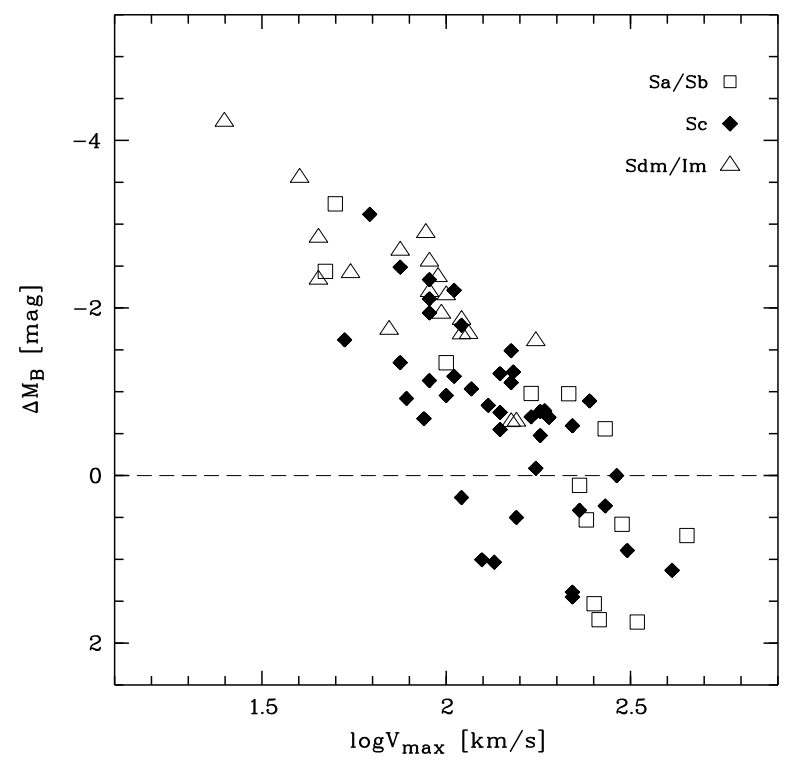

Figure 8: Offsets of the distant FORS Deep Field galaxies from the local TFR by Pierce \& Tully (1992). The distant sample is subdivided according to the SED type. All three sub-samples indicate a $\Delta M_{B}-V_{\max }$ correlation, but cover different mass regimes. See text for details.

to speculate whether this could be attributed to different criteria of rotation curve quality or differences in the $V_{\max }$ derivation procedures. On the other hand, a sample of 64 galaxies drawn from the same survey (the DEEP project, see Koo 2001) finds, in comparison to local galaxies, a tilt of the intermediateredshift luminosity-metallicity relation which is also indicated by our data (Fig. 馬).

Is is a complicated issue to determine the key processes which can give rise to the mass-dependent TF offsets we observe. Several effects likelywise act in combination when local and distant spirals are compared. E.g., the stellar populations of the intermediate-redshift galaxies are probably younger than those of their local counterparts, the gas mass fractions and chemical yields also evolve with time etc. Since even a relatively small fraction of young, high-mass stars can have a significant effect on the luminosity in the blue bands, a straightforward interpretation would be that the TF offsets of the FDF galaxies point towards a correlation between mass and age.

As a first deeper insight into this matter, Ferreras et al. (2004) have used single-zone models of chemical enrichment on a sub-sample of the FDFTF galaxies at $z>0.5$. These models were determined by only four free parameters: formation redshift, gas infall timescale, star formation efficiency and gas outflow fraction. Model star formation histories were generated which, combined with the latest Bruzual \& Charlot models (2003), were used to compute 




Figure 9: Offsets $\Delta \log r_{\mathrm{d}}$ of the distant FORS Deep Field galaxies (high quality rotation curves only) from the local velocity-size relation of the sample by Haynes et al. (1999) shown in Fig. 6 plotted as a function of redshift. Objects with $\Delta \log r_{\mathrm{d}}>0$ have larger disks than local spirals at a given $V_{\max }$, whereas values $\Delta \log r_{\mathrm{d}}<0$ correspond to disks which are smaller than in the local universe. As indicated by the fit to the data (solid line), we find a slight trend towards smaller disks at higher redshifts, in agreement with theoretical predictions within the hierarchical scenario (Mo et al. 1998, dotted line).

simulated $U B g R I J K$ broad-band colors. Probing a large volume in parameter space, these synthetic colors were fitted to the observed broad-band colors, thus deriving the four model parameters for each of the $z>0.5$ FDF galaxies. The best-fitting models indicated that high-mass galaxies on the average have higher star formation efficiencies, with a "break" at $V_{\max } \approx 140 \mathrm{~km} / \mathrm{s}$ which - for reasonable $M / L$ ratios - interestingly is good agreement with the results found by Kauffmann et al. (2003) for local galaxies from the Sloan Digital Sky Survey.

Moreover, the best-fitting formation redshifts of the Ferreras et al. models were found to be higher for the more massive FDFTF galaxies than for lowmass galaxies. The models hence yielded evidence that high-mass spirals started to convert their gas into stars at earlier cosmic epochs and on shorter timescales than low-mass ones. When evolved to zero redshift, the mean model stellar ages turned out to be older in high-mass galaxies than in lowmass galaxies. These results hint towards an anti-hierarchical evolution of the stellar (baryonic) component, a phenomenon that recently has been referred to as "down-sizing" (e.g., Kodama et al. 2004). 
It is however improbable that this implies a contradiction to the hierarchical growth of the Cold Dark Matter halos. In Fig. 9] we show the offsets $\Delta \log r_{\mathrm{d}}$ of the FDFTF galaxies with high quality rotation curves from the local velocity-size relation (which we presented in Fig. 6) as a function of redshift. Given their maximum rotation velocity, distant galaxies with $\Delta \log r_{\mathrm{d}}<0$ have disks that are smaller than in the local universe, while the disks of galaxies with $\Delta \log r_{\mathrm{d}}>0$ are larger. Though the scatter in $\Delta \log r_{\mathrm{d}}$ is substantial, we observe a slight trend towards smaller disk sizes at higher redshifts. This is in compliance with the results from other observational studies (e.g., Giallongo et al. 2000, Ferguson et al. 2004). Moreover, as is depicted in Fig. 9, the fit to the data is in relatively good agreement with the prediction of disk growth in the hierarchical scenario (e.g. Mo et al. 1998).

On the one hand, we find that the observed luminosity evolution of the FDF galaxies deviates from the results of simulations which were used to predict the TFR of distant spirals. On the other hand, the intermediateredshift disks are observed to be smaller than locally, which is in compliance with the CDM hierarchical scenario. The results from single-zone models might indicate that the star formation is suppressed in low-mass galaxies due to, e.g., SN feedback (cf. Dalcanton et al. 2004). Potentially, the discrepancies between observations and simulations arise from the fact that the mechanisms suppressing the star formation are not yet implemented realistically enough in models of galaxy evolution.

\section{Conclusions}

Using the FORS instruments of the VLT in multi-object spectroscopy mode and HST/ACS imaging, we have observed a sample 113 disk galaxies in the FORS Deep Field. The galaxies reside at redshifts $0.1<z<1.0$ and thereby probe field galaxy evolution over more than half the age of the universe. All spectrophotometric types from Sa to Sdm/Irr are comprised. Spatially resolved rotation curves have been extracted and fitted with synthetic velocity fields that account for geometric distortions as well as blurring effects arising from seeing and optical beam smearing. The intrinsic maximum rotation velocities $V_{\max }$ were derived for 73 galaxies within the field-of-view of the ACS images. Two-dimensional surface brightness profile fits were performed to measure the structural parameters like disk inclinations, position angles etc.

The massive distant galaxies fall onto the local Tully-Fisher Relation, while the low-mass distant galaxies are brighter than locally by up to $>2^{m}$ in rest-frame $B$. This trend might be combined with an evolution in metallicity. We find no evidence for a bias or systematic errors that could induce the observed shallow slope of the Tully-Fisher Relation at intermediate redshifts. Discrepancies between several previous studies could be explained as a combination of selection effects and small number statistics on the basis of such a mass-dependent luminosity evolution. On the other hand, this evolution 
would be at variance with the predictions from numerical simulations. For a given $V_{\max }$, the disks of the distant galaxies are slightly smaller than those of their local counterparts, as expected for a hierarchical structure growth. Our results therefore are discrepant with theoretical predictions only in terms of the stellar populations properties. A possible explanation could be the suppression of star formation in low-mass disks which is not yet properly implemented in models of galaxy evolution.

\section{Acknowledgements}

This study is based on observations with the European Southern Observatory Very Large Telescope (observing run IDs 65.O-0049, 66.A-0547 and 68.A-0013). We are grateful for the continuous support of our project by the PI of the FDF consortium, Prof. I. Appenzeller (LSW Heidelberg), and by Prof. K. J. Fricke (USW Göttingen). We also thank Drs. J. Heidt (LSW Heidelberg), D. Mehlert (LSW Heidelberg) and S. Noll (USW München) for performing the spectroscopic observations and ESO for the efficient support during the observations. Furthermore we want to thank J. Fliri and A. Riffeser (both USW München) for the cosmic ray rejection on the ACS images and Dr. B. Milvang-Jensen and M. Panella (both MPE Garching) for fruitful discussions. Our work was funded by the Volkswagen Foundation (I/76 520) and the Deutsches Zentrum für Luft- und Raumfahrt (50 OR 0301).

\section{References}

Bender, R., Appenzeller, I., Böhm, A., et al. 2001, The FORS Deep Field: Photometric redshifts and object classification, in Deep Fields, ed. S. Cristiani, A. Renzini, \& R. E. Williams, ESO astrophysics symposia (Springer), 96

Bertin, E., \& Arnouts, S. 1996, A\&AS, 117, 393

Boissier, S., \& Prantzos, N. 2001, MNRAS, 325, 321

Böhm, A., Ziegler, B. L., Saglia, R. P., et al. 2004, A\&A, 420, 97

Bruzual, A. G., \& Charlot, S. 2003, MNRAS, 344, 100

de Jong, R. S. 1996, A\&A, 313, 377

Dalcanton, J. J., Yoachim, P., \& Bernstein, R. A. 2004, ApJ, 608, 189

Ferguson, H. C. F., Dickinson, M., Giavalisco, M., et al. 2004, ApJ, 600, L107

Ferreras, I., \& Silk, J. 2001, ApJ, 557, 165

Ferreras, I., \& Silk, J., Böhm, A., \& Ziegler, B. L. 2004, MNRAS, 355, 64

Giallongo, E., Menci, N., Poli, F., et al. 2000 ApJ, 530, L73

Giovanelli, R., Haynes, M. P., Herter, T., et al. 1997, AJ, 113, 53

Governato, F., Mayer, L., Wadsley, J., et al. 2004, ApJ, 607, 688

Haynes, M. P., Giovanelli, R., Chamaraux, P., et al. 1999, AJ, 117, 2039

Heidt, J., Appenzeller, I., Gabasch, A., et al. 2003, A\&A, 398, 49

Kannappan, S. J., Fabricant, D. G., \& Franx, M. 2002, AJ, 123, 2358

Kauffmann, G., Heckman, T. M., White, S. D. M., et al. 2003, MNRAS, 341, 54 
Kobulnicky, H. A., Willmer, C. N. A., Phillips, A. C., et al. 2003, ApJ, 599, 1006

Kodama, T., Yamada, T., Akiyama, M., et al. 2004, MNRAS, 350, 1005

Koo, D. C. 2001, DEEP: Pre-DEIMOS Surveys to I 24 of Galaxy Evolution and Kinematics, in Deep Fields, ed. S. Cristiani, A. Renzini, \& R. E. Williams, ESO astrophysics symposia (Springer), 107

Lambas, D. G., Tissera, P. B., Sol Alonso, M., \& Coldwell, G. 2003, MNRAS, 346, 1189

Mathewson, D. S., \& Ford, V. L. 1996, ApJS, 107, 97

McGaugh, S. 1991, ApJ, 380, 140

Milvang-Jensen, B., Aragón-Salamanca, A., Hau, G. K. T., Jørgensen, I., \& Hjorth, J. 2003, MNRAS, 339, L1

Mo, H. J., Mao, S., \& White, S. D. M. 1998, MNRAS, 295, 319

Navarro, J. F., \& White, S. D. M. 1994, MNRAS, 267, 401

Noll, S., Mehlert, D., Appenzeller, I., et al. 2004, A\&A, 418, 885

Peacock, J. A. 2003, RSPTA, 3671, 2479

Peng, C. Y., Ho, L. C., Impey, C. D., \& Rix, H.-W. 2002, AJ, 124, 266

Persic, M., \& Salucci, P., \& Stel, F. 1996, MNARS, 281, 27

Pierce, M. J., \& Tully, R. B. 1992, ApJ, 387, 47

Rix, H.-W., Guhathakurta, P., Colless, M., \& Ing, K. 1997, MNRAS, 285, 779

Sakai, S., Mould, J. R., Hughes, S. M. G., et al. 2000, ApJ, 529, 698

Simard, L., \& Pritchet, C. J. 1998, ApJ, 505, 96

Sofue, Y. \& Rubin, V. 2001, ARA\&A, 39, 137

Spergel, D. N., Verde, L., Peiris, H. V., et al. 2003, ApJS, 148, 175

Steinmetz, M., \& Navarro, J. F. 1999, ApJ, 513, 555

Tully, R. B., \& Fisher, J. R. 1977, A\&A, 54, 661

Tully, R. B., \& Fouqué, P. 1985, ApJS, 58, 67

Tully, R. B., Pierce, M. J., Huang, J.-S., et al. 1998, AJ, 115, 2264

van den Bosch, F. 2002, MNRAS, 332, 456

Verheijen, M. A. W. 2001, ApJ, 563, 694

Vogt, N. P., Forbes, D. A., Phillips, A. C., et al. 1996, ApJ, 465, L15

Vogt, N. P., Phillips, A. C., Faber, S. M., et al. 1997, ApJ, 479, L121

Vogt, N. P. 2001, Distant Disk Galaxies: Kinematics and Evolution to z 1, in Deep Fields, ed. S. Cristiani, A. Renzini, \& R. E. Williams, ESO astrophysics symposia (Springer), 112

Ziegler, B. L., Böhm, A., Fricke, K. J., et al. 2002, ApJ, 564, L69 